\title{
Selective EEG analysis for emotion recognition using multidimensional directed information criteria
}

\author{
Panagiotis Petrantonakis, Vagia Kaltsa, Leontios Hadjileontiadis \\ From $1^{\text {st }}$ International Congress on Neurobiology and Clinical Psychopharmacology \\ and European Psychiatric Association Conference on Treatment Guidance \\ Thessaloniki, Greece. 19-22 November 2009
}

\section{Background}

Brain waves captured by multiple electrodes during an electroencephalogram (EEG) recording, are derived as time series signals that represent the brain activity of various scalp sites. One important feature of these multiple time series is the information flow from one to another that under specific processing can reveal useful brain functionality.

\section{Materials and methods}

In this work, the Multidimensional Directed Information (MDI) [1] concept is adopted in order to examine the flow of information between EEG recordings from three different scalp sites with the objective to bring out and define the interconnections of different scalp sites of frontal and prefrontal cortex during an emotionally charged situation. In the line of these objectives, EEG signals were recorded from 16 healthy right-handed subjects during a specifically designed emotion elicitation experiment. Two monopole and one dipole EEG channels were placed at Fp1, Fp2 and F3/F4 positions respectively, according to the international 10/20 system [2].

\section{Results}

The EEG signals were analyzed using MDI as a parameter that could identify the EEG sections that contribute the most to the emotion recognition. In this way, an efficient emotion categorization could be achieved by keeping the effective parts of the EEG signal and further analyze it feeding a classifier towards the development of a robust and effective emotion recognition system from EEG recordings.

\section{Conclusions}

The encouraging preliminary results justify the feasibility of the proposed approach, stressing the importance of the targeted selection of the information source within the EEG recordings before any further categorization analysis.

\section{Acknowledgements \\ The authors would like to thank all the 16 subjects participated in the experiment for their patience during the tedious EEG recording phase.}

Published: 22 April 2010

\section{References}

1. Sakata O, Shiina T, Saito Y: Multidimensional directed information and its application. Electronics and Communication in Japan 2002, 85:45-55.

2. Jasper $\mathrm{H}$ : The ten-twenty electrode system of the International Federation 1958. Electroencephalogr Clin Neurophysiol 10:367-380.

\section{doi:10.1186/1744-859X-9-S1-S149}

Cite this article as: Petrantonakis et al: Selective EEG analysis for emotion recognition using multidimensional directed information criteria. Annals of General Psychiatry 2010 9(Suppl 1):S149. 